\title{
Dietary supplementation with Bifidobacterium longum subsp. infantis ( $B$. infantis) in healthy breastfed infants: study protocol for a randomised controlled trial
}

Smita Awasthi ${ }^{1}$, Reason Wilken ${ }^{1}$, Forum Patel ${ }^{1}$, J. Bruce German², David A. Mills ${ }^{2}$, Carlito B. Lebrilla ${ }^{3}$, Kyoungmi Kim ${ }^{4}$, Samara L. Freeman ${ }^{5}$, Jennifer T. Smilowitz ${ }^{5}$, April W. Armstrong ${ }^{6}$ and Emanual Maverakis ${ }^{1 *}$

\begin{abstract}
Background: The development of probiotics as therapies to cure or prevent disease lags far behind that of other investigational medications. Rigorously designed phase I clinical trials are nearly non-existent in the field of probiotic research, which is a contributing factor to this disparity. As a consequence, how to appropriately dose probiotics to study their efficacy is unknown. Herein we propose a novel phase I ascending dose trial of Bifidobacterium longum subsp. infantis (B. infantis) to identify the dose required to produce predominant gut colonisation in healthy breastfed infants at 6 weeks of age.

Methods/design: This is a parallel-group, placebo-controlled, randomised, double-blind ascending dose phase I clinical trial of dietary supplementation with $B$. infantis in healthy breastfed infants. The objective is to determine the pharmacologically effective dose (ED) of $B$. infantis required to produce predominant ( $>50 \%$ ) gut colonisation in breastfed infants at 6 weeks of age. Successively enrolled infant groups will be randomised to receive two doses of either B. infantis or placebo on days 7 and 14 of life. Stool samples will be used to characterise the gut microbiota at increasing doses of $B$. infantis.

Discussion: Probiotic supplementation has shown promising results for the treatment of a variety of ailments, but evidence-based dosing regimes are currently lacking. The ultimate goal of this trial is to establish a recommended starting dose of $B$. infantis for further efficacy-testing phase II trials designed to evaluate $B$. infantis for the prevention of atopic dermatitis and food allergies in at-risk children.
\end{abstract}

Trial registration: Clinicaltrials.gov \#NCT02286999, date of trial registration 23 October 2014.

Keywords: Bifidobacterium infantis, Probiotic, Atopy, Dose-escalation, Phase I clinical trial, Atopic dermatitis, Food allergy

\section{Background}

The idea of probiotics, "live microorganisms that when administered in adequate amounts confer a health benefit on the host", dates back to Elie Metchnikoff who hypothesised over 100 years ago that lactic acid bacilli had health benefits [1]. To date, thousands of reports of probiotics have supported the potential health benefits

\footnotetext{
*Correspondence: emaverakis@ucdavis.edu

'Department of Dermatology, University of California Davis Medical Center,

Sacramento, CA, USA

Full list of author information is available at the end of the article
}

of these bacteria and the World Health Organization (WHO) has formulated guidelines for their use as nutritional supplements as well as investigation into their potential therapeutic properties [2]. Probiotics have been studied for the treatment and prevention of a variety of diseases in children including atopic dermatitis, bacterial gastroenteritis, inflammatory bowel disease, and necrotizing enterocolitis [3-5]. Overall, the data concerning probiotics as preventive agents for atopic diseases such as atopic dermatitis and food allergies are inconclusive,

\section{Biomed Central}

(c) 2016 Awasthi et al. Open Access This article is distributed under the terms of the Creative Commons Attribution 4.0 International License (http://creativecommons.org/licenses/by/4.0/), which permits unrestricted use, distribution, and reproduction in any medium, provided you give appropriate credit to the original author(s) and the source, provide a link to the Creative Commons license, and indicate if changes were made. The Creative Commons Public Domain Dedication waiver (http://creativecommons.org/publicdomain/zero/1.0/) applies to the data made available in this article, unless otherwise stated. 
with some studies suggesting a possible benefit and others showing mixed results $[4,6-8]$.

Humans and other mammals are hosts to a diverse set of symbiotic as well as pathogenic intestinal bacteria. Approximately 1000 microbial species reside at a density of $1 \times 10^{12}$ organisms per gram of colonic content $[9,10]$. Several studies have demonstrated a clear link between immune system development and the composition of gut microbiota [11-13]. In addition, disruption of intestinal barrier function may lead to premature exposure of the infant to atopy-inducing environmental allergens, which would theoretically predispose the infant to the development of food allergies [14]. By protecting against colonisation by pathogenic bacteria, probiotics may help protect intestinal barrier function and thus decrease the susceptibility for development of atopic diseases and food allergies [15]. However, the pharmacokinetics of commensal bacteria and the dosage required to achieve predominant gut colonisation has thus far not been quantified. The probiotic that will be administered in our proposed phase I clinical trial, Bifidobacterium longum subsp. infantis (B. infantis), is unique in that it is able to fully utilise human milk oligosaccharides [16]. Thus, an exclusively breastfed infant's gut provides the ideal environment to facilitate colonisation with this commensal bacteria [16-18].

In order to proceed with designing a rigorous phase II clinical trial program to evaluate B. infantis as a preventative measure for a variety of childhood ailments, including atopic disease, it is necessary to first determine the pharmacologically effective dose (ED) of $B$. infantis. To date, pharmacologically guided phase I studies have not been conducted in the field of probiotics. We are thus proposing a phase I dose-escalation trial to evaluate the safety and pharmacokinetics of $B$. infantis supplementation when administered to healthy breastfed infants. The primary endpoint of the proposed trial is identification of the ED of $B$. infantis, defined as the dose required to produce predominant $(>50 \%)$ gastrointestinal colonisation in breastfed infants by 6 weeks of age. The $50 \%$ value was chosen based on the proportion of $B$. infantis colonisation observed in breastfed infants in less-developed countries that have a low incidence of atopic disease compared to infants in the United States, specifically that seen in Bangladeshi infants $[19,20]$.

If successful, this study will be the first of its kind to establish a standardised dosing regime for probiotic supplementation in infants and will serve as a platform from which to design phase II and III trials to investigate the ability of $B$. infantis to protect against the development of a variety of childhood illnesses, including atopic dermatitis and food allergies.

\section{Methods/Design}

\section{Study design}

The proposed phase I clinical trial is a parallel-group, placebo-controlled, randomised, double-blind ascending dose study of dietary supplementation with $B$. infantis in healthy breastfed infants to evaluate its safety as well as determine the ED of $B$. infantis producing $>50 \%$ gut colonisation at 6 weeks of age. Infants will be enrolled sequentially in groups of five (three randomised to receive $B$. infantis and two to receive placebo). The trial participants and investigators will be blinded as to their group randomisation, which will be conducted by the study pharmacist (who will then dispense blinded $B$. infantis or placebo to the investigators). Depending on group assignment, each infant will receive one dose of either B. infantis or placebo on day 7 and another on day 14 of life (two doses total). For infants in the $B$. infantis group, a calculated maximally recommended starting dose (MRSD) will be used to initiate the dose escalation and is defined below. Every 2 weeks, an additional group of five infants (randomised 3:2 to B. infantis and placebo) will be enrolled to receive progressively higher doses of $B$. infantis. Calculation of the appropriate dose escalation will be performed using a modified Fibonacci series as described below in an effort to identify the ED of B. infantis.

After the ED of $B$. infantis has been identified (defined as the dose capable of producing $50 \%$ gut colonisation by 6 weeks of age) two additional sequential dose escalations will be performed. The purpose of the final two dose escalations is to determine if successively higher doses of $B$. infantis result in increased gut colonisation or barrier protection, or, alternatively, if a maximum effective dose (MaxED) for B. infantis exists above which there is no further increase in gut colonisation or barrier protection. Following the final dose escalation, Hanley's Rule of Three will be applied in order to determine if lower-frequency adverse events are caused by $B$. infantis. Hanley's Rule of Three states that in order to identify any adverse events occurring at a frequency of 1:10 or greater with a $95 \%$ confidence interval, at least 30 subjects must be enrolled [21]. A schematic overview of the trial design and dose escalation protocol is provided in Fig. 1.

Study visits will be scheduled for weeks 1, 2, 6, 24, 36, 52, and 78. Parents will complete surveys at each study visit to monitor the infants for potential adverse events associated with probiotic administration including feeding intolerance, fevers, or bowel irregularities including constipation and diarrhoea.

Stool samples will be collected twice weekly for the first 6 weeks of life then once weekly at weeks 24, 36, 52 , and 78. Stool samples will be analysed to determine the relative abundance of $B$. infantis over time, and the overall diversity of the gut microbiota with and without 


\section{Phase I Ascending Dose Study}

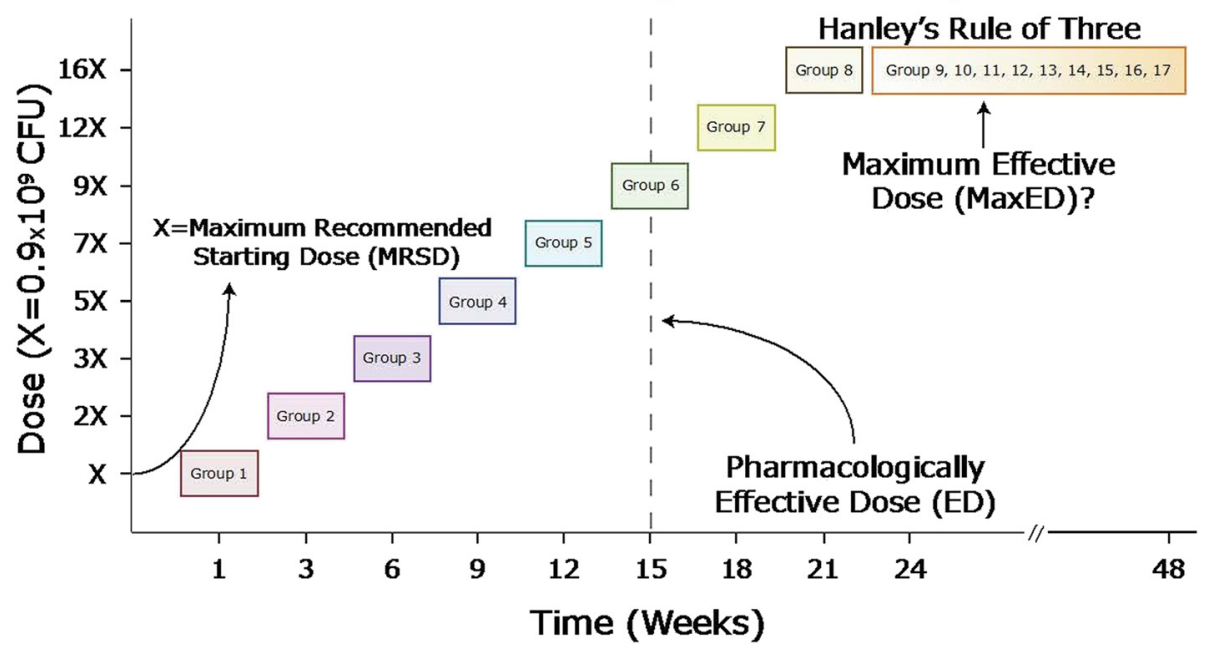

Fig. 1 A parallel-group, placebo-controlled, randomised, double-blind ascending dose phase I study of dietary supplementation with B. infantis. Groups of healthy infants will receive increasing doses of $B$. infantis until it comprises $50 \%$ of their gut microbiota, defined as the pharmacologically effective dose (ED). This figure arbitrarily designates Group 6 as receiving the ED of $B$. infantis. After the ED has been reached, two additional dose escalations will occur to determine the impact that additional $B$. infantis has on the gut microbiota. To satisfy Hanley's Rule of Three, 30 infants will receive the highest dose of $B$. infantis. " $X$ " represents the maximum recommended starting dose (MRSD). A modified Fibonacci Series ( $X, 2 X, 3 X, \ldots)$ is used to guide the dose escalations. CFU colony-forming units

B. infantis supplementation. Stools will also be analysed for milk oligosaccharides to verify consumption of breast milk and to correlate the proportion of human milk oligosaccharides and free sugar monomers seen in the infant stool at various levels of $B$. infantis colonisation.

Breast milk will be collected at 7, 14, 42, 120, and 180 days after the birth of the infant to determine the type and proportion of milk oligosaccharides present at various time points. The purpose of this analysis is to allow correlation of the type and amount of milk glycans present in breast milk with that in the infant stool samples.

Entry into the study requires the intent to breastfeed exclusively for a minimum of 6 months. If mothers decide to discontinue breastfeeding during the study, we will note that in the infant's notes and obtain an additional series of weekly stool samples for 6 weeks after discontinuation of breastfeeding. The purpose of this additional stool sample collection is to determine if discontinuation of breastfeeding has an impact on the level of existing $B$. infantis colonisation in the infant gut.

A table of the schedule of study visits and specimen collections is provided in Table 1, and an overview of the timeline of the study interventions and assessments is provided in Table 2 .

\section{Patient recruitment}

As infants need to be enrolled within the first week of life, efforts will target pregnant women. Institutional Review Board (IRB)-approved flyers will be used to recruit pregnant women to participate in the study from the UC Davis Family Practice Center, UC Davis Obstetrics \& Gynecology offices, and the inpatient Labor and Delivery ward.

\section{Informed consent process}

During the initial screening visit, written informed consent will be obtained from the mother for infant participation in the study. The parents will be instructed on the proper collection and home storage of infant stool and maternal breast milk samples.

The potential risks and benefits to enrolled infants are outlined below in lay terms, as described in the IRB-approved consent forms that will be used to consent the parents/guardians of eligible infants for study participation:

"Probiotics are living organisms such as bacteria or yeast that are sold as dietary supplements for the purpose of improving health. Probiotics may have benefits such as decreasing the growth of harmful bacteria in the gastrointestinal tract, improving digestion and helping to strengthen the immune system. Probiotics have shown some benefit in reducing the risk of developing atopic (allergic) dermatitis in children. However, this optimal dose of probiotic needed to achieve such beneficial effects has not yet been studied in randomised controlled clinical trials. We wish to investigate the optimal dose of probiotic bacteria needed to colonise the infant gastrointestinal tracts by 6 weeks of age. We will also 
Table 1 Schedule of study visits and specimen collections for each infant and mother

\begin{tabular}{|c|c|}
\hline Week & Visits and activities \\
\hline Prior to starting the study & $\begin{array}{l}\text { - The patients' parents or legal guardians will be asked to read and sign the consent form } \\
\text { and will be instructed on the procedures for collection and storage of stool samples. They } \\
\text { will be asked to refrain from giving the child any other dietary supplementation or probiotics } \\
\text { while in the study and to inform the researchers if they are prescribed oral antibiotics at } \\
\text { any time during the study. }\end{array}$ \\
\hline \multirow[t]{4}{*}{ Week 1} & $\begin{array}{l}\text { - Initial study visit, adverse event survey administration to parents and administration of } \\
\text { first dose of } B \text {. infantis or placebo on day } 7 \text { of life. }\end{array}$ \\
\hline & $\begin{array}{l}\text { - Week } 1 \text { stool samples collected (infants will have stool samples collected twice weekly } \\
\text { for the first } 6 \text { weeks and then at the study visits at weeks } 6,24,36,52 \text {, and } 78 \text { ). }\end{array}$ \\
\hline & $\begin{array}{l}\text { - If breastfeeding is discontinued at any point in the study, an additional series of six weekly } \\
\text { stool samples will be collected following discontinuation in addition to the regularly } \\
\text { scheduled collections }\end{array}$ \\
\hline & - Mother collects breast milk on day 7 of life. \\
\hline \multirow[t]{3}{*}{ Week 2} & $\begin{array}{l}\text { - Study visit, adverse event survey administration to parents and administration of second } \\
\text { dose of } B \text {. infantis or placebo on day } 14 \text { of life. }\end{array}$ \\
\hline & -Week 2 stool samples collected \\
\hline & - Mother collects breast milk on day 14 of life. \\
\hline \multirow[t]{2}{*}{ Week 3} & - Week 3 stool samples collected \\
\hline & - Adverse event survey administration to parents \\
\hline \multirow[t]{2}{*}{ Week 4} & -Week 4 stool samples collected \\
\hline & - Adverse event survey administration to parents \\
\hline Week 5 & -Week 5 stool samples collected \\
\hline \multirow[t]{3}{*}{ Week 6} & - Study visit, adverse event survey administration to parents. \\
\hline & - Week 6 stool sample collected. \\
\hline & - Mother collects breast milk on day 42 of life. \\
\hline Week 17 & - Mother collects breast milk on day 120 of life. \\
\hline \multirow[t]{2}{*}{ Week 24} & - Study visit, adverse event survey administration to parents. \\
\hline & - Week 24 stool sample collected. \\
\hline Week 25 & - Mother collects breast milk on day 180 of life. \\
\hline \multirow[t]{2}{*}{ Week 36} & - Study visit, adverse event survey administration to parents. \\
\hline & -Week 36 stool sample collected. \\
\hline \multirow[t]{2}{*}{ Week 52} & - Study visit, adverse event survey administration to parents. \\
\hline & -Week 52 stool sample collected. \\
\hline \multirow[t]{2}{*}{ Week 78} & - Study visit, adverse event survey administration to parents. \\
\hline & - Week 78 stool sample collected. \\
\hline
\end{tabular}

monitor patients for any evidence of atopic dermatitis (AD) and any effect on the severity of the AD during the course of the study.

Side effects described with probiotic use include diarrhea, vomiting and increased flatulence. Serious adverse effects of probiotics in infants include an extremely low risk of systemic infection (bacteraemia), but have thus far only been reported in children who were immunocompromised. Only healthy infants without any major systemic illnesses will be eligible to participate in this study, so we anticipate very minimal physical risks to subjects under these conditions.
However, as a result of being in the study your infant may experience one or more of the following adverse side effects listed below:

- Fever greater than $102^{\circ}$ Farenheit

- Feeding difficulties (decreased feeding, colic, spitting up)

- Irregular bowel movements (diarrhea or constipation)

- Blood or pus in the stool

- Vomiting

- Abdominal pain or swelling 
Table 2 Schedules for study participant enrolment, interventions and assessments in parallel-group, placebo-controlled, randomised, double-blind ascending dose phase I study of dietary supplementation with B. infantis

\begin{tabular}{|c|c|c|c|c|c|c|c|c|c|c|c|c|c|c|}
\hline \multirow[b]{2}{*}{ Timepoint } & \multirow{2}{*}{$\begin{array}{l}\text { Enrolment } \\
-\mathrm{t}_{1}\end{array}$} & \multirow{2}{*}{$\begin{array}{l}\text { Allocation } \\
0\end{array}$} & \multicolumn{12}{|c|}{ Post-allocation } \\
\hline & & & Week 1 & Week 2 & Week 3 & Week 4 & Week 5 & Week 6 & Week 17 & Week 24 & Week 25 & Week 36 & Week 52 & Week 78 \\
\hline \multicolumn{15}{|l|}{ Enrolment: } \\
\hline Eligibility screen & $x$ & & & & & & & & & & & & & \\
\hline Informed consent & $x$ & & & & & & & & & & & & & \\
\hline Allocation & & $x$ & & & & & & & & & & & & \\
\hline \multicolumn{15}{|l|}{ Interventions: } \\
\hline Dose \#1 of B. infantis/placebo & & & $x$ & & & & & & & & & & & \\
\hline Dose \#2 of B. infantis/placebo & & & & $x$ & & & & & & & & & & \\
\hline Infant stool collection & & & $x$ & $x$ & $x$ & $x$ & $x$ & $x$ & & $x$ & & $x$ & $x$ & $x$ \\
\hline Breast milk collection & & & $\begin{array}{l}\text { X } \\
\text { (Day } 7 \\
\text { of life) }\end{array}$ & $\begin{array}{l}\text { X } \\
\text { (Day } 14 \\
\text { of life) }\end{array}$ & & & & $\begin{array}{l}X \\
\text { (Day } 42 \\
\text { of life) }\end{array}$ & $\begin{array}{l}\text { X } \\
\text { (Day 120 } \\
\text { of life) }\end{array}$ & & $\begin{array}{l}\text { X } \\
\text { (Day } 180 \\
\text { of life) }\end{array}$ & & & \\
\hline \multicolumn{15}{|l|}{ Assessments: } \\
\hline $\begin{array}{l}\text { Identification of pharmacologically } \\
\text { effective dose (ED) of } B \text {. infantis }\end{array}$ & & & & & & & & $x$ & & & & & & \\
\hline Study visit & & & $x$ & $x$ & & & & $x$ & & $x$ & & $x$ & $x$ & $x$ \\
\hline Adverse event survey & & & $x$ & $x$ & & & & $x$ & & $x$ & & $x$ & $x$ & $x$ \\
\hline Analysis of stool oligosaccharides & & & $x$ & $x$ & $x$ & $x$ & $x$ & $x$ & & $x$ & & $x$ & $x$ & $x$ \\
\hline Analysis of stool microbiota & & & $x$ & $x$ & $x$ & $x$ & $x$ & $x$ & & $x$ & & $x$ & $x$ & $x$ \\
\hline $\begin{array}{l}\text { Analysis of breast milk } \\
\text { oligosaccharides }\end{array}$ & & & $\begin{array}{l}\text { X } \\
\text { (Day } 7 \\
\text { of life) }\end{array}$ & $\begin{array}{l}\text { X } \\
\text { (Day } 14 \\
\text { of life) }\end{array}$ & & & & $\begin{array}{l}X \\
\text { (Day } 42 \\
\text { of life) }\end{array}$ & $\begin{array}{l}\text { X } \\
\text { (Day } 120 \\
\text { of life) }\end{array}$ & & $\begin{array}{l}\text { X } \\
\text { (Day } 180 \\
\text { of life) }\end{array}$ & & & \\
\hline
\end{tabular}


- Sepsis (serious infection involving the presence of bacteria in the baby's blood)"

\section{Study inclusion and exclusion criteria Inclusion criteria}

- Healthy newborn infants between 1 and 7 days old with intent to be exclusively breastfed for a minimum of 6 months

\section{Exclusion criteria}

- Infants given dietary supplementation, including other probiotics.

- Infants born prior to 34 weeks gestation.

- Infants below $10^{\text {th }}$ percentile for body weight.

- Postnatal use of antibiotics (oral, intramuscular, or intravenous) by either the mother or the infant. Of note, prenatal maternal Group B streptococcus prophylaxis is not a criterion for study exclusion.

- Family history of immunodeficiency syndrome(s).

- Infants with signs of a clinically apparent underlying immunodeficiency.

- Intent to use non-breast milk infant formula for feeding during the first 6 months.

- History of gastrointestinal tract abnormality or infection.

\section{Protocol for calculating the starting dose of $B$. infantis and dose escalation}

Previous studies of B. infantis supplementation in premature infants at the UC Davis neonatal intensive care unit (NICU) have used a dose of $1.4 \times 10^{9}$ colonyforming units twice daily for 2 weeks without any observed serious adverse effects [22]. Applying a safety factor of three and adjusting for once-daily dosing yields, a MSRD of $0.9 \times 10^{9}$ colony-forming units of $B$. infantis is to be administered on day 7 and day 14 of life. Each group of five infants will be enrolled at least 2 weeks apart to allow for sufficient time to identify possible adverse events prior to each dose increase. A modified Fibonacci-based dose escalation protocol will be applied as shown below:

$$
F_{n}=\sum_{k=0}^{\left[\frac{n-1}{2}\right]}\left(\begin{array}{c}
n-k-1 \\
k
\end{array}\right)
$$

The specific modified-Fibonacci series will be as follows:

$\times, 2 \times, 3 \times, 5 \times, 9 \times, 12 \times$, and $16 \times\left(\right.$ where $\times=0.9 \times 10^{9}$ colony-forming units of $B$. infantis)

Based on the schedule for enrolling new groups every 2 weeks, by the time the pharmacologically effective dose (ED, defined as the dose of $B$. infantis resulting in $50 \%$ gut colonisation at 6 weeks of age) has been identified, two additional dose escalations will have been performed in successive infant groups. Figure 1 provides schematic representation of infant group enrollment as well as the dose escalation protocol to identify the ED for B. infantis as well as the MaxED, if applicable.

\section{Study-wide number of subjects}

We propose a maximum sample size of 90 infants. Five infants (three to receive $B$. infantis supplementation and two to receive placebo) will be enrolled for the initial dose of B. infantis. An additional five infants will be enrolled at each dose escalation. Once the pharmacologically effective dose (ED) of $B$. infantis has been reached, two additional dose escalations will be performed (for reasons as described above). We estimate that the ED of $B$. infantis will be identified within seven dose escalations (35 infants). Accounting for the final two dose escalations will bring the total to 45 infants. Following the final dose escalation, 45 additional infants (27 to receive B. infantis and 18 to receive placebo) will be enrolled to screen for lower-frequency adverse events using Hanley's Rule of Three [21]. This will bring the maximum enrollment number to 90 infants. If the ED of B. infantis required to produce $50 \%$ gut colonisation at 6 weeks is not reached, then enrollment will be halted after 90 infants have been enrolled. Additional stopping rules are listed in the "Study Endpoints" section.

\section{Randomisation}

A computer-generated list of random numbers will be used to create a series of numbered, sealed opaque envelopes containing assignments to either placebo or supplementation with $B$. infantis. For every three infants assigned to $B$. infantis supplementation, two additional infants will be assigned to placebo. The study pharmacist will be responsible for the randomisation and delivery of the blinded supplements.

\section{Study methods and interventions}

\section{Infant stool collection}

Parents will be instructed how to collect and store infant stool samples at home. Stool samples will be collected twice weekly for the first 6 weeks of life then once weekly at weeks $24,36,52$, and 78 . If breastfeeding is discontinued at any point, an additional series of weekly stool samples will be collected for 6 weeks following discontinuation. At the initial enrollment visit parents will be provided with a stool sample collection including a sealable freezer box, marked sealed collection tubes and sealable plastic bags. Parents will be instructed to store samples in their home freezers immediately after collection to prevent secondary bacterial growth, and discard 
any samples not able to be frozen at the time of collection. Stool samples will be stored in the home freezers of study participants and picked up by study personnel once weekly to ensure they can be properly transported to the laboratory under temperature-controlled conditions. Any samples that are not stored immediately in the freezer at the time of collection or thaw en route to the laboratory will be discarded and parents will be instructed to collect and store a new stool sample according to the protocol. The stool samples will be analysed in the laboratory using DNA extraction to determine the identity and predominance of various commensal bacterial species, including the percentage of B. infantis present. The method of stool collection that will be provided to parents is described below:

1. At any time of day, scoop 1-2 teaspoons of your infant's stool with the tongue depressor into the sealable collection tube. The amount of stool should fill the tube between the 5 and $10 \mathrm{ml}$ mark.

2. Seal the tube and label with the time and date it was collected.

3. Place the stool filled tube inside a sealable plastic bag and seal it.

4. IMMEDIATELY place the sealed bag containing the stool sample into the study freezer box and store it in your freezer. It is important that stool samples are stored in the freezer immediately after collection to prevent bacteria from growing. If the stool sample was not immediately placed in the freezer, please discard the sample and collect another sample from your baby at the earliest convenience.

\section{Breast milk collections}

Mothers will collect a series of breast milk samples for laboratory analysis to determine the type and proportion of milk oligosaccharides present at various time points. The purpose of this analysis is to allow correlation of the type and amount of milk glycans present in breast milk with that in the infant stool samples, both to verify the presence of the specific oligosaccharides preferred by $B$. infantis as well as to determine if appropriate amounts are present in the infant stool to represent exclusive breastfeeding practices.

Breast milk will be collected at 7, 14, 42, 120, and 180 days after the birth of the infant, and stored in the home freezers in the same manner as the stool samples. Parents will be provided with detailed instructions and supplies for collection as described below:

1. Between 2-4 hours after your last breastfeeding, use the breast pump to pump all the milk from one breast into the breast pump collection bottle.
2. Using the measuring cup, measure out 12 ounces of breast milk.

3. Divide the 12 ounces of breast milk into three (3) collection tubes, with four (4) ounces per tube.

4. Using the collection tube labels and a permanent marker, label each collection tube with the date and time of collection.

5. IMMEDIATELY, place the three tubes of breast milk in a single ziplock bag, seal it, and place in the study collection box stored in your freezer.

6. Wash and dry the breast pump, collection bottle, and measuring cup thoroughly after each use

\section{Data management and specimen banking}

Collected breast milk and stool samples will be labelled with a four digit number randomly assigned to each patient as well as the date of sample collection. De-identified samples will be delivered to the laboratory for processing. Precautions will be taken to maintain the privacy of all participants. Personal information maintained on infant subjects will include age, first initial, last name, and four digit medical record number assigned at the time of randomisation. Patient information will not be disclosed to third party individuals except those authorised to oversee the research project.

\section{Study endpoints}

Primary outcome measures

Identification of pharmacologically effective dose (ED) of $B$. infantis

The primary endpoint of the study is identification of the ED of $B$. infantis, i.e. the dose required to produce predominant $(>50 \%)$ gut colonisation at 6 weeks of age. The value of $50 \%$ colonisation was chosen because $B$. infantis represented greater than $50 \%$ of the gut microbiota in the vast majority of Bangladesh infants [19]. The percent gut colonisation will be determined through analysis of stool samples by $16 \mathrm{~S}$ sequencing.

\section{Safety}

An additional primary endpoint is to determine the safety of B. infantis supplementation in immunocompetent, fullterm infants. Any adverse events including fever of $38.9^{\circ} \mathrm{C}$ $\left(102^{\circ} \mathrm{F}\right)$ or higher, abdominal pain or colic, blood or purulence in the stool, diarrhoea or vomiting will be documented and dosing adjusted accordingly ("Stopping rules" are defined below).

\section{Secondary outcome measures Milk oligosaccharide consumption}

All stool samples will also be analyzed for the presence of oligosaccharides unique to breast milk and for the presence of free saccharide monomers, which are 
products of their incomplete digestion. B. infantis abundance will be correlated to these values.

\section{Microbiota composition}

In addition to determining the percent composition of $B$. infantis in infant stool samples, numerous other measures such as microbiota diversity (Shannon Diversity plots) and rate of $B$. infantis decline following cessation of breast feeding will also be determined.

\section{Provisions to monitor data and ensure safety of subjects}

Children will be recruited for this study. Subjects will be monitored clinically during the study period to assess for potential adverse events. A previous study of $B$. infantis supplementation in premature infants administered similar doses and was well tolerated with no severe adverse events noted [22]. Nonetheless, patients enrolled in the study will be monitored closely and any evidence of feeding intolerance, illness, or infection will be thoroughly evaluated. A survey to assess for any baseline feeding intolerance or symptoms will be administered to all infants prior to administration of $B$. infantis or placebo. Additional symptom questionnaires will be administered weekly during the first month of the study and at every study visit therafter at $6,24,36,52$, and 78 weeks. Patients will be provided with the contact information for the principal investigator and on-call dermatology resident should any unforeseen symptoms or problems arise.

\section{Rules for stopping dose escalation and halting trial}

1) Dose escalation will be stopped if a maximally tolerated dose (MTD) of B. infantis is reached. The data safety monitoring board will evaluate the adverse events reported at each ascending dose of $B$. infantis. If the safety monitoring board deems that one or more of the three infants in the $B$. infantis supplementation group experienced an adverse event (such as fever $>38.9^{\circ} \mathrm{C}\left(102^{\circ} \mathrm{F}\right)$, abdominal pain or colic, blood or purulence in the stool, diarrhoea or vomiting) then additional dose escalation will be halted.

2) In the event dose escalation is halted due to adverse events, an additional group of five infants will be enrolled with three infants at the same (i.e. not escalated) dose of $B$. infantis and two receiving placebo.

3) If no adverse events occur in this additional, nonescalated group, then dose escalation will be resumed beginning with the next group of enrolled infants.

4) However, if adverse events are noted in one or more infant(s) in the non-escalated group, all further dose escalation will be halted. The B. infantis dose will be de-escalated to the previous value, and an additional group of five infants (three $B$. infantis, two placebo) will be enrolled. If no adverse events occur in the de-escalated group, this dose will be considered the MTD and the trial will be stopped.

5) If any adverse events are noted in the de-escalated group, further de-escalation and enrollment of infants will occur to identify the dose of $B$. infantis considered to be the MTD, in which no adverse events occur in the study group.

\section{Withdrawal of subjects}

Subjects may be withdrawn without their consent if they acquire medical issues during the study period that require administration of oral or parenteral antibiotics or immunosuppressive medications. If a subject is to be withdrawn, the parent(s) will be contacted and the reason(s) for withdrawal will be explained in full. No further data will be collected from withdrawn subjects.

Subjects have the right to withdraw from the study at any time for any reason. Every effort will be made to follow-up subjects who discontinue placebo or B. infantis supplementation prior to the second dose. These evaluations should continue according to the protocol of scheduled study visits if at all possible. The reasons for discontinuation will be recorded in the subjects' study file. If a subject is unable to return for evaluation, every effort will be made to contact via telephone 28 days after withdrawal to determine if any serious adverse events have occurred while off study. Any identified adverse events will be followed until resolution.

The investigator also has the right to remove subjects from the study without their consent. Possible reasons for removal include:

- Non-compliance with the study protocol

- Significant protocol deviation

- Serious adverse event potentially related to study treatment

Subjects that withdraw after the 6 week study visit will not be replaced with new subjects.

\section{Discussion}

From our experience of administering B. infantis to infants in the NICU at UC Davis, it is highly unlikely that a phase I trial will successfully identify a MTD of this probiotic. Thus, our proposed phase I ascending dose study will instead utilise a targeted outcome, optimal gut colonisation of B. infantis at 6 weeks of age. Such "concentration controlled" phase I clinical trials that use pharmacokinetics in place of drug toxicity to guide dose escalation are supported in the literature [23, 24]. For example, in molecularly targeted anticancer agents, toxicity concerns are reduced, making "drug-related biological effects" a better suited primary endpoint [25-28]. 
Our specific trial will measure gut microbiota composition following $B$. infantis administration to determine the dose of $B$. infantis that is required to successfully colonise the gastrointestinal tract of a 6-week-old breastfed infant. Secondary outcome measures will include measurement of complex glycan consumption by the infant as well as diversity of the intestinal microbiota. As in all phase I studies, the infants will be monitored closely for adverse events. The main goal of this phase I trial is to establish a recommended administering dose of $B$. infantis to support further efficacy testing in phase II and phase III trials.

Prior probiotic studies have differed from the proposed study with regards to their dosing regime, inclusion criteria, and types of bacteria administered. Many prior trials in infants were designed with limited knowledge of the human milk glycome and resultant effects on the infant's intestinal microbiotia. Probiotic bacteria used in prior trials in children have included Bifidobacterium breve, Bifidobacterium lactis, Bifidobacterium longum, Lactobacillus acidophilus, Lactobacillus fermentum, and Propinobacterium freudenreichii, among others [29-34]. In many prior studies, it was unclear why a particular probiotic was chosen. Research done by the milk group at UC Davis has shown that the vast majority of probiotic species do not grow on the human milk oligosaccharides in breast milk [16, 35, 36]. Evidently, most probiotics studied in previous clinical trials have been selected based on culturability and taste profiles when administered as fermented food products rather than their biological activity. In contrast, our proposed study is based upon research into the human milk glycome and insight into the specific bacteria that digest breast milk oligosaccharides.

One unique aspect of this trial is that we will administer $B$. infantis to exclusively breastfed infants. The breast milk will provide the prebiotic oligosaccharides and thus select for the growth of $B$. infantis over other gastrointestinal commensal bacteria. In addition, we will monitor the composition of the intestinal microbiota should the mothers choose to discontinue breastfeeding to determine the effect breast milk consumption has on the maintenance of $B$. infantis intestinal colonisation. Previous studies investigating probiotics have administered the bacteria in combination with galacto- and fructo-oligosaccharides $[37,38]$. However, these sugars differ significantly from milk oligosaccharides in that they are linear rather than branched and lack fucose and sialic acid moieties [39]. As human breast milk will comprise the majority of an infant's diet, supplementing with a probiotic that thrives on human milk oligosaccharides may be the most sensible strategy for cultivating a protective microbiota in healthy infants.
The most common adverse effects described with probiotic use include diarrhoea, vomiting, and increased flatulence. Probiotics are known to be extremely safe, as evidenced by prior use in premature infants as well as in both adults and children with HIV [40, 41]. In Finland, the use of probiotic supplements (namely Lactobacillus rhamnosous GG) has increased dramatically over the past 20 years without evidence of a corresponding increase in the rate of lactobacillus bactermia [42, 43]. Probiotic supplementation has also been shown to be well tolerated without adverse effects in a recent randomised, double-blind, placebo-controlled trial comparing a combination of lactobacilli (Lactobacillus salivarius and Lactobacillus paracasei) and bifidobacteria (Bifidobacterium animalis subsp. lactis and Bifidobacterium bifidum) in pregnant women and infants, with the goal of assessing the safety of probiotic supplementation [44]. In this trial, enrolled women were treated with a total of $1 \times 10^{9}$ colony-forming units of this probiotic regime daily for the last month of pregnancy and the regime was then administered to their infants from birth through to 6 months of age. There was no significant difference in the incidence of adverse events in either the maternal or infant groups, and no adverse events were attributed directly to the probiotic supplementation. There have been rare reports demonstrating the possibility of probiotic-related infectious complications, such as sepsis, bacteraemia, and endocarditis [45-47]. However, it is estimated that the risk of developing bacteraemia from ingested Lactobacillus probiotics is less than one in one million [48]. Though extremely rare, sepsis due to bifidobacterium has been described [49-51]. The described cases of bifidobacterium bacteraemia include one adult that developed incidental sepsis following acupuncture, and two infants that received Bifidobacterium breve or Bifidobacterium longum as a probiotic supplement. Of note, one infant was premature with extremely low birthweight and the other was full term with comorbid omphalocele. To date there are no reported instances of bacteraemia resulting from $B$. infantis; however, all study participants will be warned of the extremely low risk of such complications.

While studies have demonstrated the ability of certain probiotics to prevent childhood illnesses [8], there are virtually no data on how to appropriately dose these supplements. The typical probiotic study has adopted a daily dosing regime, making feasibility an issue. Sometimes the probiotic is administered to the mother first and then to the infant for the first 6 months of life. Dose-ranging studies are usually required early on during a drug's clinical development. Excluding a few exceptions, probiotic dose-ranging studies are for the most part non-existent [52-54]. As a follow-up clinical trial, we plan to conduct a dose-ranging phase II study that 
will compare the effectiveness of $B$. infantis daily dosing to a limited dosing regime consisting of $B$. infantis administered on days 7 and 14 only. In theory, the human breast milk oligosaccharides will bestow $B$. infantis with a competitive growth advantage, making daily dosing unnecessary. If the limited dosing regime is successful, it will have an immediate health care impact, as inoculating breast fed infants with two doses of $B$. infantis is an extremely feasible preventative measure to reduce the incidence of atopy and other diseases in at-risk children.

The pharmacokinetically guided design of this proposed phase I trial has not been attempted in prior probiotic studies. If successful the trial will identify a pharmacologically effective dose (ED) and maximum effective dose (MaxED) for B. infantis supplementation in healthy infants. The next generation sequencing strategy that will be employed to analyse the stool of the infants to determine gut colonisation with $B$. infantis has been well standardised, and is a common method for analysis of gut microbiota. Infants will also be monitored closely for potential adverse effects in case a maximally tolerated dose (MTD) is reached during the ascending dose study. The design of the trial is also innovative because the specific probiotic used for the supplementation will have a competitive advantage in an exclusively breastfed infant; it is the only bacterium that can fully utilise the complex oligosaccharides in breast milk as an energy source. Thus, the design of the trial provides an optimal environment for $B$. infantis to outcompete other intestinal microbiota. The rationale for the choice of probiotic used in prior infant trials is not clear; however, in this study it is based upon knowledge of the human milk glycome and specific metabolic needs of $B$. infantis. Our ultimate goal is to develop a clinically appropriate, safe, and effective dosing regime for probiotics that may be utilised in further phase II and phase III clinical trials. In doing so, we hope to lay the foundation for the design of objective, standardised clinical trials to assess the efficacy of probiotics for the prevention of a variety of diseases, including atopic dermatitis and food allergies in at-risk infants.

\section{Trial status}

This protocol is for a proposed clinical trial. The phase I protocol has been reviewed and approved by the UC Davis Institutional Review Board (IRB). This trial has been registered through clinicaltrials.gov on 23 October 2014 and is accessible online (Identification Number NCT02286999). Patient recruitment has not yet commenced for this trial.

\section{Abbreviations}

B. infantis, Bifidobacterium longum subsp. infantis; ED, pharmacologically effective dose; MaxED, maximum effective dose; MSRD, maximally recommended starting dose; MTD, maximally tolerated dose; NICU, neonatal intensive care unit

\section{Acknowledgements}

The authors are funded by the Burroughs Wellcome Fund.

\section{Authors' contributions}

SA participated in the design of the protocol and drafting of the manuscript. RW participated in drafting of the manuscript and design of the protocol. FP participated in drafting of the manuscript. JBG participated in conceptualisation and design of the study protocol. DAM participated in the design of the protocol. CBL participated in the design of the protocol, with specific focus on the sample analysis methods. KK contributed to the design of the statistical aspects of the protocol. SLF contributed to the protocol design, specifically the visit schedule and sample collection methods. JTS contributed to the design of the protocol, specifically the visit schedule and sample collection methods. AWA contributed to the design of the protocol. EM participated in the conceptualisation of the study, protocol design, and drafting of the manuscript. All authors read and approved the final manuscript.

\section{Competing interests}

The authors declare that they have no competing interests.

\section{Ethics approval and consent to participate}

A review of the protocol by the University of California Davis IRB has been conducted and the protocol was approved on 22 October 2015.

\section{Author details}

${ }^{1}$ Department of Dermatology, University of California Davis Medical Center, Sacramento, CA, USA. ${ }^{2}$ Department of Food Science \& Technology, University of California Davis, Davis, CA, USA. ${ }^{3}$ Department of Chemistry, University of California Davis, Davis, CA, USA. ${ }^{4}$ Division of Biostatistics, Department of Public Health Sciences, University of California Davis, Davis, CA, USA. ${ }^{5}$ Foods for Health Institute, University of California Davis, Davis, CA, USA.

${ }^{6}$ Department of Dermatology, Keck School of Medicine at the University of Southern California, Los Angeles, CA, USA.

Received: 24 December 2015 Accepted: 2 July 2016

Published online: 22 July 2016

\section{References}

1. Mackowiak PA. Recycling Metchnikoff: probiotics, the intestinal microbiome and the quest for long life. Front Public Health. 2013;1:52.

2. Reid $\mathrm{G}$, et al. The importance of guidelines in the development and application of probiotics. Curr Pharm Des. 2005;11(1):11-6.

3. Viljanen $\mathrm{M}$, et al. Probiotics in the treatment of atopic eczema/dermatitis syndrome in infants: a double-blind placebo-controlled trial. Allergy. 2005; 60(4):494-500.

4. Rautava S, Kalliomaki M, Isolauri E. Probiotics during pregnancy and breastfeeding might confer immunomodulatory protection against atopic disease in the infant. J Allergy Clin Immunol. 2002;109(1):119-21.

5. Weston $\mathrm{S}$, et al. Effects of probiotics on atopic dermatitis: a randomised controlled trial. Arch Dis Child. 2005;90(9):892-7.

6. Abrahamsson TR, et al. Probiotics in prevention of IgE-associated eczema: a double-blind, randomised, placebo-controlled trial. J Allergy Clin Immunol. 2007;119(5):1174-80

7. Rautava S, Arvilommi H, Isolauri E. Specific probiotics in enhancing maturation of IgA responses in formula-fed infants. Pediatr Res. 2006;60(2):221-4.

8. Kalliomaki $\mathrm{M}$, et al. Probiotics in primary prevention of atopic disease: a randomised placebo-controlled trial. Lancet. 2001;357(9262):1076-9.

9. Hooper LV, et al. Molecular analysis of commensal host-microbial relationships in the intestine. Science. 2001;291(5505):881-4.

10. Hooper LV, Gordon JI. Commensal host-bacterial relationships in the gut. Science. 2001;292(5519):1115-8.

11. Mazmanian SK, et al. An immunomodulatory molecule of symbiotic bacteria directs maturation of the host immune system. Cell. 2005;122(1):107-18.

12. Rakoff-Nahoum S, et al. Recognition of commensal microflora by toll-like receptors is required for intestinal homeostasis. Cell. 2004;118(2):229-41.

13. Mazmanian SK. Capsular polysaccharides of symbiotic bacteria modulate immune responses during experimental colitis. J Pediatr Gastroenterol Nutr. 2008:46 Suppl 1:E11-2.

14. Yu LC. Intestinal epithelial barrier dysfunction in food hypersensitivity. J Allergy (Cairo). 2012;2012:596081. 
15. Cao S, Feehley TJ, Nagler CR. The role of commensal bacteria in the regulation of sensitization to food allergens. FEBS Lett. 2014;588(22):425866.

16. German JB, et al. Human milk oligosaccharides: evolution, structures and bioselectivity as substrates for intestinal bacteria. Nestle Nutr Workshop Ser Pediatr Program. 2008;62:205-18. discussion 218-22.

17. Barboza M, et al. Glycoprofiling bifidobacterial consumption of galactooligosaccharides by mass spectrometry reveals strain-specific, preferential consumption of glycans. Appl Environ Microbiol. 2009;75(23):7319-25.

18. LoCascio RG, et al. Glycoprofiling of bifidobacterial consumption of human milk oligosaccharides demonstrates strain specific, preferential consumption of small chain glycans secreted in early human lactation. J Agric Food Chem. 2007;55(22):8914-9.

19. Huda MN, et al. Stool microbiota and vaccine responses of infants. Pediatrics. 2014;134(2):e362-72

20. Spergel JM. Epidemiology of atopic dermatitis and atopic march in children Immunol Allergy Clin North Am. 2010;30(3):269-80.

21. Hanley JA, Lippman-Hand A. If nothing goes wrong, is everything all right? Interpreting zero numerators. JAMA. 1983;249(13):1743-5.

22. Underwood MA, et al. A comparison of two probiotic strains of bifidobacteria in premature infants. J Pediatr. 2013;163(6):1585-91. e9.

23. Le Tourneau C, Lee JJ, Siu LL. Dose escalation methods in phase I cancer clinical trials. J Natl Cancer Inst. 2009;101(10):708-20.

24. LoRusso PM, Boerner SA, Seymour L. An overview of the optimal planning, design, and conduct of phase I studies of new therapeutics. Clin Cancer Res. 2010;16(6):1710-8.

25. Korn EL, et al. Clinical trial designs for cytostatic agents: are new approaches needed? J Clin Oncol. 2001;19(1):265-72.

26. Parulekar WR, Eisenhauer EA. Phase I trial design for solid tumor studies of targeted, non-cytotoxic agents: theory and practice. J Natl Cancer Inst. 2004; 96(13):990-7.

27. Sleijfer S, Wiemer E. Dose selection in phase I studies: why we should always go for the top. J Clin Oncol. 2008;26(10):1576-8.

28. Cannistra SA. Challenges and pitfalls of combining targeted agents in phase I studies. J Clin Oncol. 2008;26(22):3665-7.

29. Enomoto $T$ et al. Effects of bifidobacterial supplementation to pregnant women and infants in the prevention of allergy development in infants and on fecal microbiota. Allergol Int. 2014:63(4):575-85.

30. Garaiova I, et al. Probiotics and vitamin C for the prevention of respiratory tract infections in children attending preschool: a randomised controlled pilot study. Eur J Clin Nutr. 2014;69(3):373-9.

31. Forsberg $A$, et al. Pre- and postnatal administration of Lactobacillus reuteri decreases TLR2 responses in infants. Clin Transl Allergy. 2014;4:21.

32. Zampieri $\mathrm{N}$, et al. Lactobacillus paracasei subsp. paracasei $\mathrm{F} 19$ in Bell's stage 2 of necrotizing enterocolitis. Minerva Pediatr. 2013;65(4):353-60.

33. Allen SJ, et al. Probiotics in the prevention of eczema: a randomised controlled trial. Arch Dis Child. 2014:99(11):1014-9.

34. Saraoui T, et al. A unique in vivo experimental approach reveals metabolic adaptation of the probiotic Propionibacterium freudenreichii to the colon environment. BMC Genomics. 2013;14:911.

35. Sela DA, et al. The genome sequence of Bifidobacterium longum subsp. infantis reveals adaptations for milk utilization within the infant microbiome Proc Natl Acad Sci U S A. 2008;105(48):18964-9.

36. Marcobal A, et al. Consumption of human milk oligosaccharides by gutrelated microbes. J Agric Food Chem. 2010;58(9):5334-40.

37. Wu KG, Li TH, Peng HJ. Lactobacillus salivarius plus fructo-oligosaccharide is superior to fructo-oligosaccharide alone for treating children with moderate to severe atopic dermatitis: a double-blind, randomised, clinical trial of efficacy and safety. Br J Dermatol. 2012;166(1):129-36.

38. Kukkonen $\mathrm{K}$, et al. Probiotics and prebiotic galacto-oligosaccharides in the prevention of allergic diseases: a randomised, double-blind, placebocontrolled trial. J Allergy Clin Immunol. 2007;119(1):192-8.

39. Zivkovic AM, Barile D. Bovine milk as a source of functional oligosaccharides for improving human health. Adv Nutr. 2011;2(3):284-9.

40. Bernardeau $M$, et al. Safety assessment of dairy microorganisms: the Lactobacillus genus. Int J Food Microbiol. 2008;126(3):278-85.

41. Snydman DR. The safety of probiotics. Clin Infect Dis. 2008;46 Suppl 2:S104-11. discussion S144-51.

42. Saxelin M, et al. Lactobacilli and bacteremia in southern Finland, 1989-1992. Clin Infect Dis. 1996;22(3):564-6.
43. Salminen MK, et al. Lactobacillus bacteremia during a rapid increase in probiotic use of Lactobacillus rhamnosus GG in Finland. Clin Infect Dis. 2002;35(10):1155-60

44. Allen SJ, et al. Dietary supplementation with lactobacilli and bifidobacteria is well tolerated and not associated with adverse events during late pregnancy and early infancy. J Nutr. 2010;140(3):483-8.

45. Mackay $A D$, et al. Lactobacillus endocarditis caused by a probiotic organism. Clin Microbiol Infect. 1999;5(5):290-2.

46. Land $\mathrm{MH}$, et al. Lactobacillus sepsis associated with probiotic therapy. Pediatrics. 2005;115(1):178-81.

47. Rautio M, et al. Liver abscess due to a Lactobacillus rhamnosus strain indistinguishable from L. rhamnosus strain GG. Clin Infect Dis. 1999;28(5): 1159-60.

48. Borriello SP, et al. Safety of probiotics that contain lactobacilli or bifidobacteria. Clin Infect Dis. 2003:36(6):775-80.

49. Ha GY, et al. Case of sepsis caused by Bifidobacterium longum. J Clin Microbiol. 1999;37(4):1227-8.

50. Ohishi A, et al. Bifidobacterium septicemia associated with postoperative probiotic therapy in a neonate with omphalocele. J Pediatr. 2010;156(4): 679-81.

51. Jenke $A$, et al. Bifidobacterium septicaemia in an extremely low-birthweight infant under probiotic therapy. Arch Dis Child Fetal Neonatal Ed. 2012;97(3): F217-8.

52. Tsangalis $\mathrm{D}$, et al. Bioavailability of isoflavone phytoestrogens in postmenopausal women consuming soya milk fermented with probiotic bifidobacteria. Br J Nutr. 2005;93(6):867-77.

53. Lammers KM, et al. Effect of probiotic strains on interleukin 8 production by HT29/19A cells. Am J Gastroenterol. 2002;97(5):1182-6.

54. Gao XW, et al. Dose-response efficacy of a proprietary probiotic formula of Lactobacillus acidophilus CL1285 and Lactobacillus casei LBC80R for antibiotic-associated diarrhea and Clostridium difficile-associated diarrhea prophylaxis in adult patients. Am J Gastroenterol. 2010;105(7):1636-41.

\section{Submit your next manuscript to BioMed Central and we will help you at every step:}

- We accept pre-submission inquiries

- Our selector tool helps you to find the most relevant journal

- We provide round the clock customer support

- Convenient online submission

- Thorough peer review

- Inclusion in PubMed and all major indexing services

- Maximum visibility for your research

Submit your manuscript at www.biomedcentral.com/submit 